\title{
تغير مسار العالم من استخدام الطاقة النووية إلى توظيف الطاقة المتجددة
}

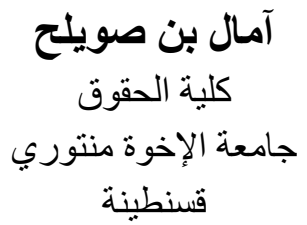

في هذا المقال نتطرق لموضوع احتل اهتمام

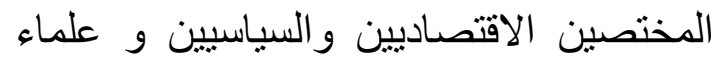

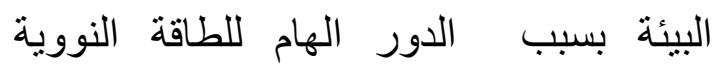

و الطاقة النظيفة في تحسين حياة الإنسان

وتطوير حياة الأجيال المستقبلية بتوفير كله

الحلول التي يمكنها جعل الحياة سهلة ومستقرة

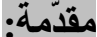

شكل موضوع اكتشاف

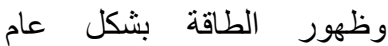

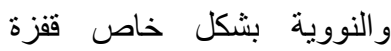

علمية نوعية نظرا للمواصفات

التي تمتاز بها هذه الطاقة , حيث

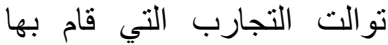
العماء الألمان في البداية لتأتي

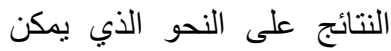

للإنسان توظيفها في مجالات

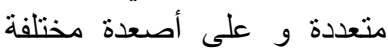

سلمية كانت أم حربية ماساهم أمعنم

\section{Abstract:}

In this article we address the subject occupied the attention of specialists economists politicians and ecologists because of the important role of nuclear power and clean energy in improving the lives of human and the development of future generations to provide all the solutions that enable them to make life easy and stable . 


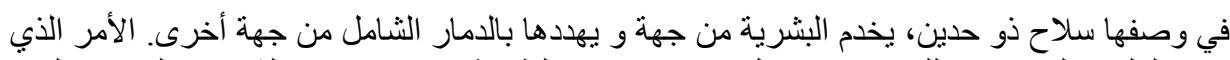

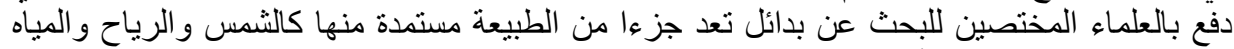

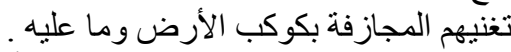

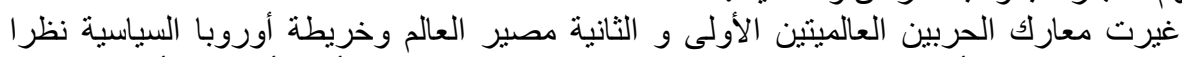

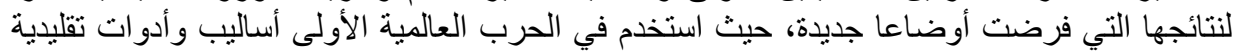

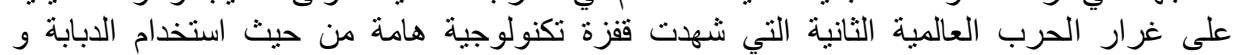

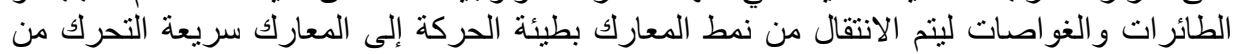

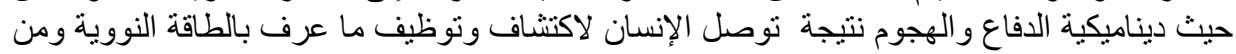

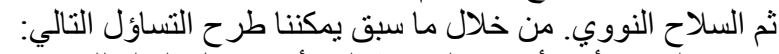

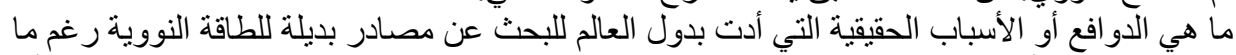

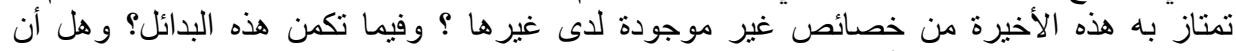

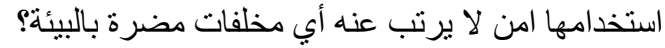

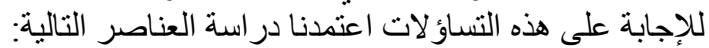

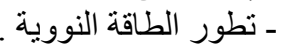
ـ ـ الاستخدامات المتنو عة للطاقة النووية و الآثار السلبية الناجمة عنها.

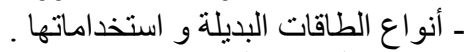

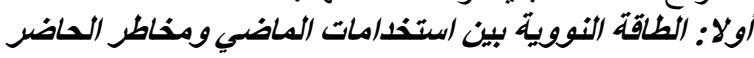

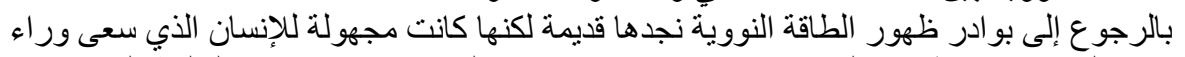

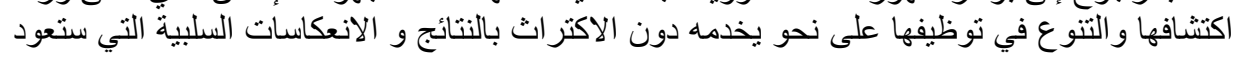

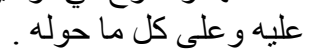
1- اكتثافت الطاقة النووية : الطاقة النووية أو الذرية اكتثاف قديم ظهر مع بداية التفكير في المادة و

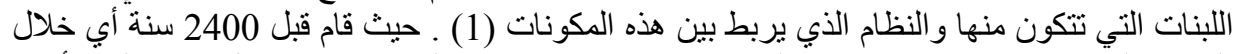

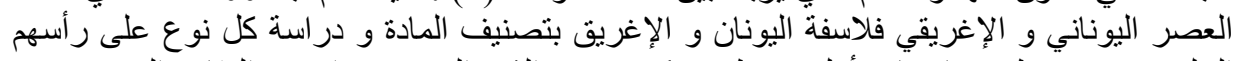

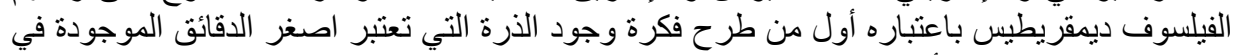

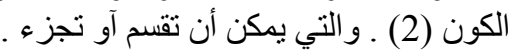

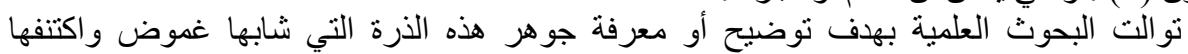

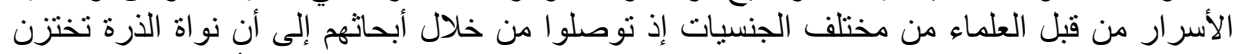

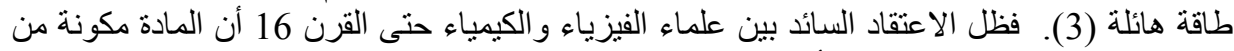

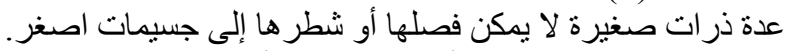

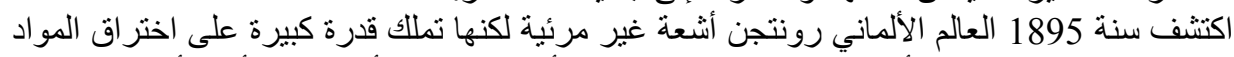

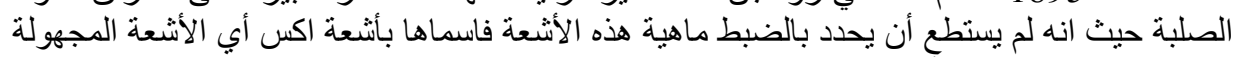

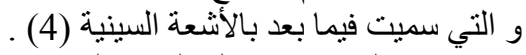

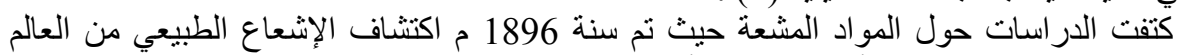

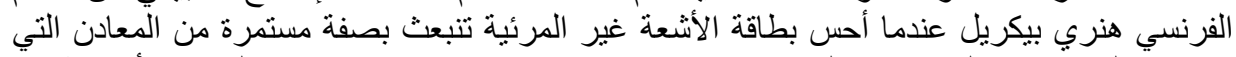

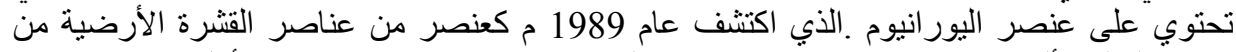

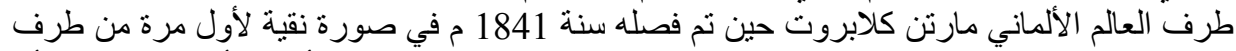

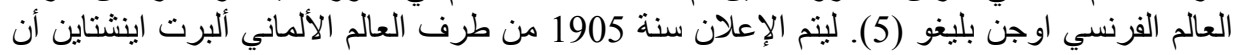

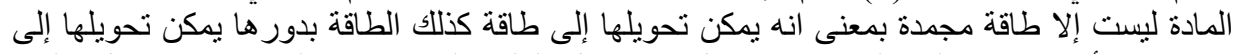

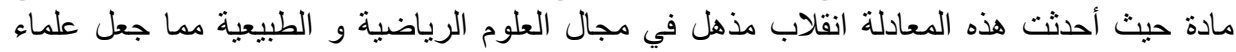

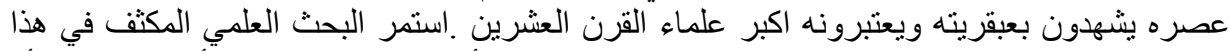

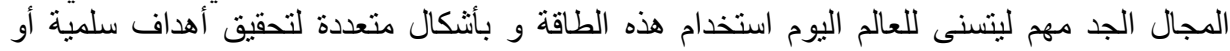

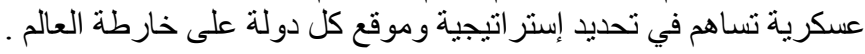


2- الاستخدامات المتعددة للطاقة النووية : وظف الإنسان الطاقة النووية في مجالات عدة وسخر ها

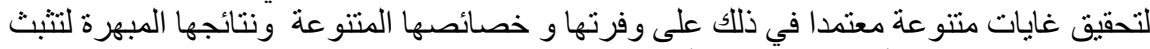

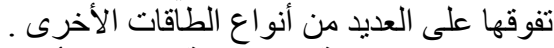

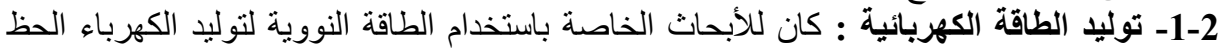

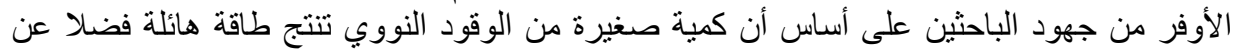

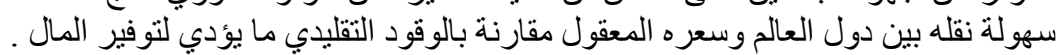

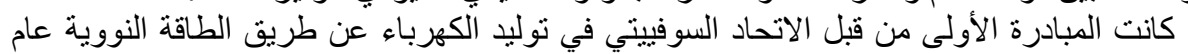

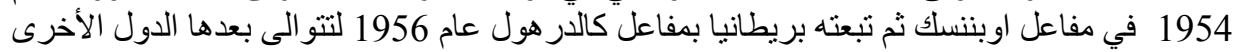

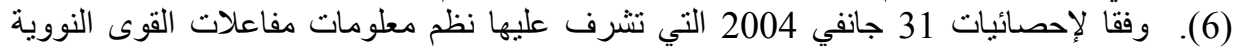

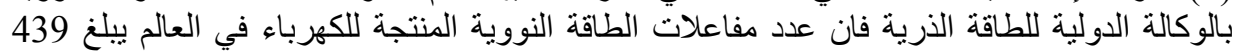

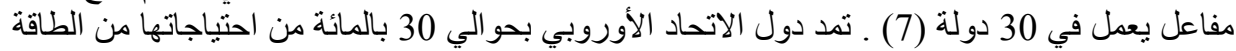

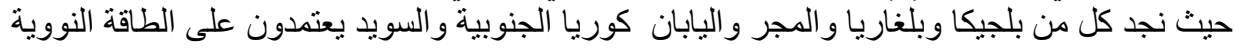

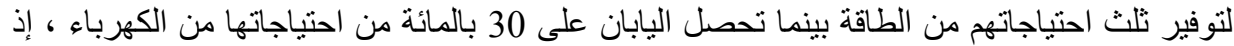

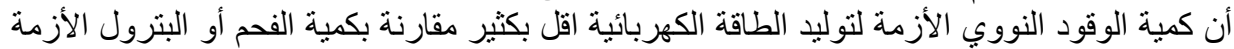

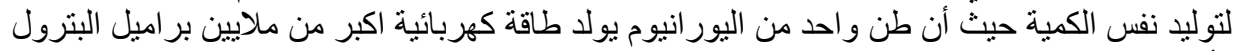
و أطنان الفحم الفئر

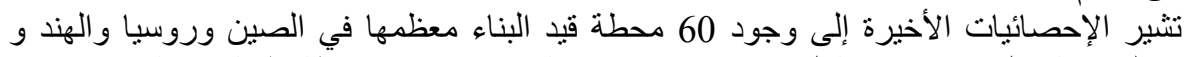

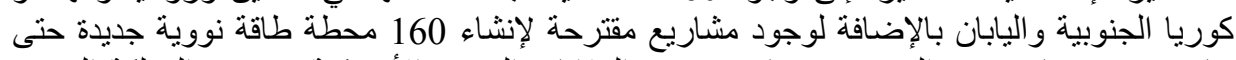

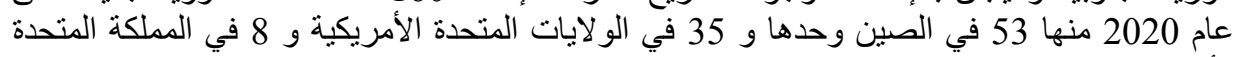

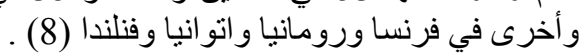

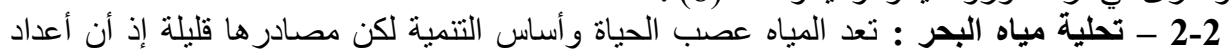

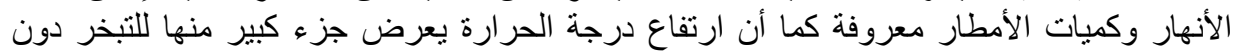

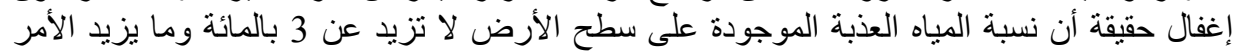

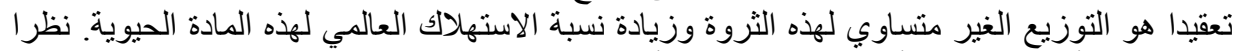

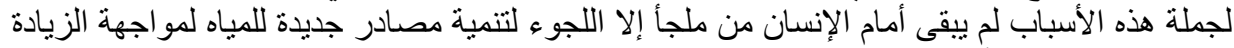

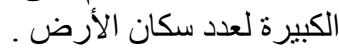

باعتبار أن مياه البحر هي مصدر لانهائي من المياه أدى للتفكير في تسخير الطاقة النووية لتحليتها

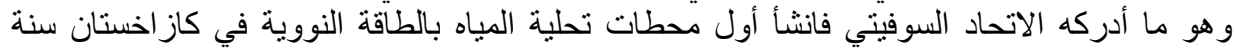

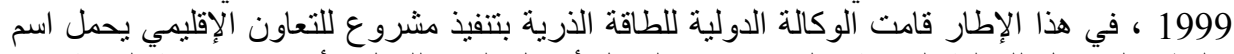

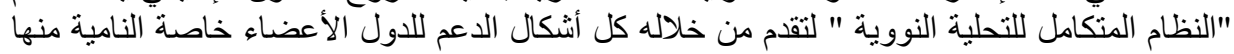

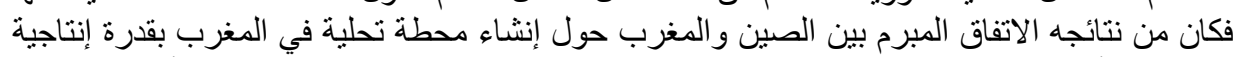

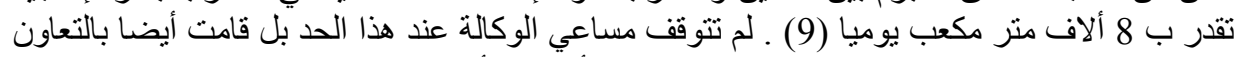

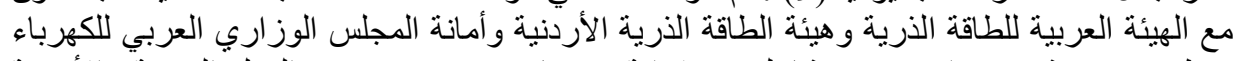

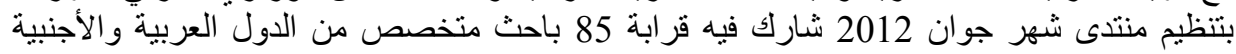
و عدد من المنظمات العربية و الدولية حيث تمحورت فئر فعاليات المنتدى على على المحاور التالية :

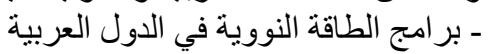

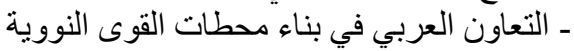

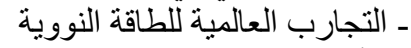

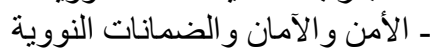

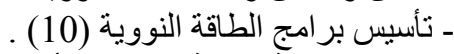

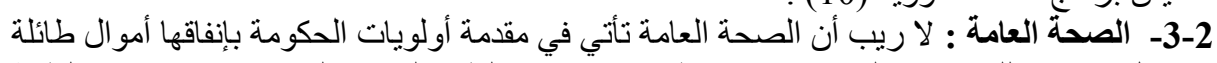

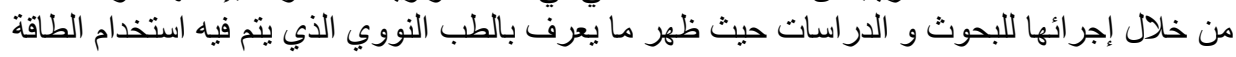




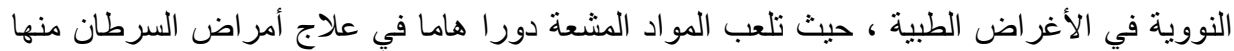

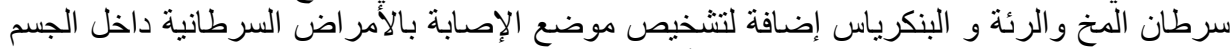

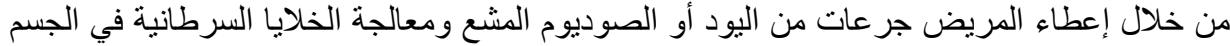

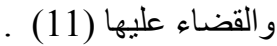

4-2-2 مجالات الزراعة والعمران : بلغت نسبة تلف المحاصيل الغذائية العالمية حوالي 35 بالمائة

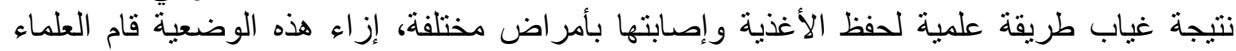

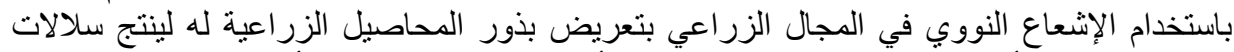

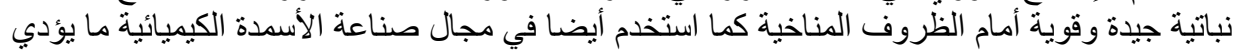

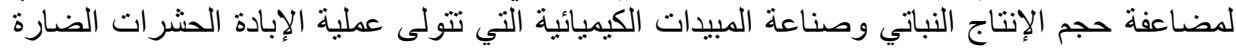

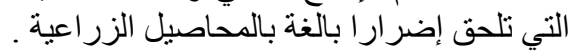

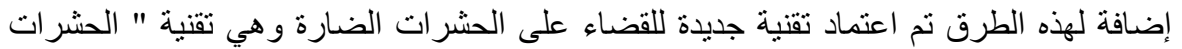

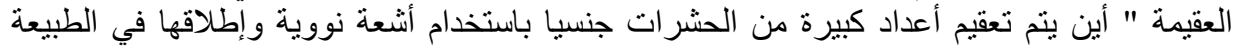

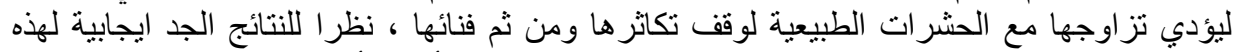

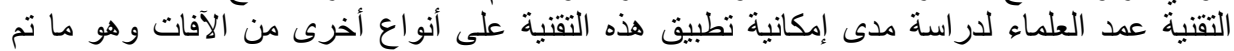

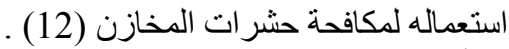

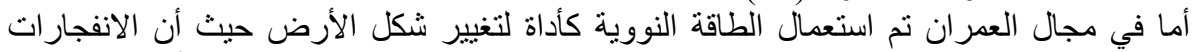

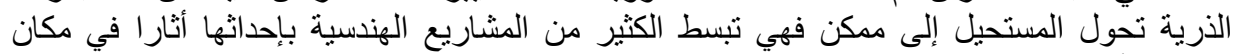

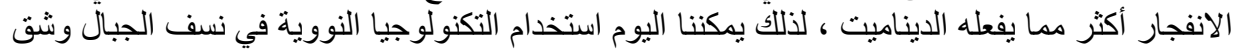

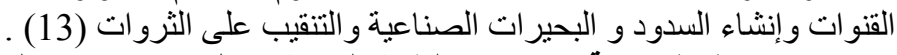

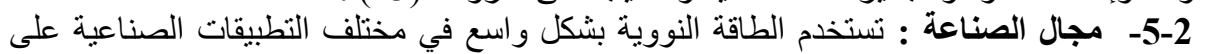

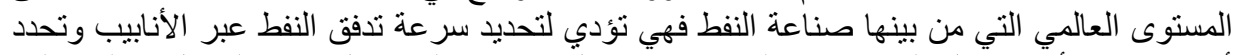

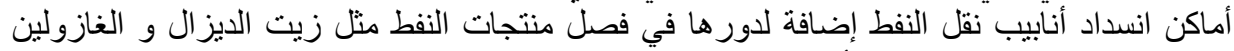

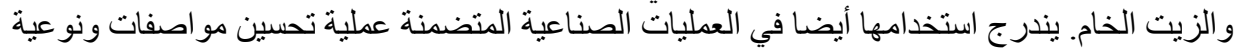

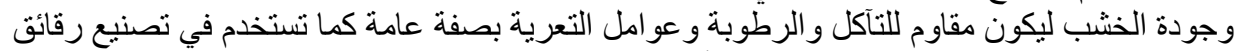

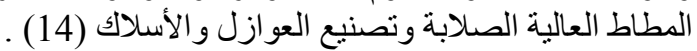

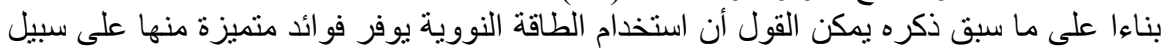

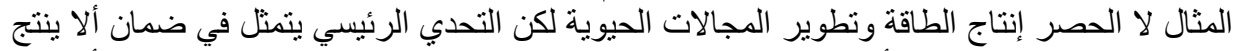

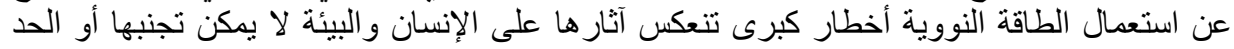

3ـ الآثار السلبية الناجمة عن توظيف الطاقة النووية : بالرجوع إلى النى النتائج الناجمة عن استخدام

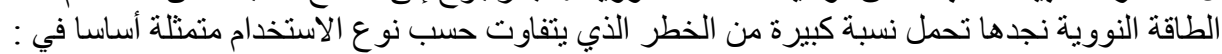

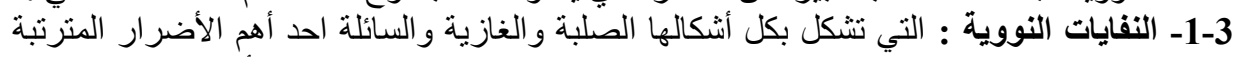

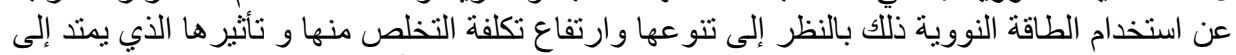

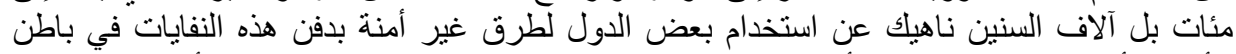

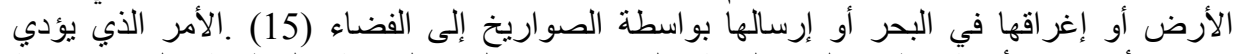

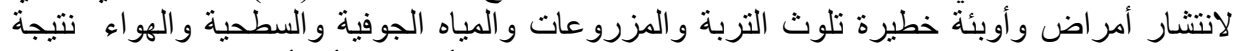

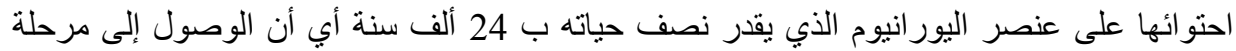

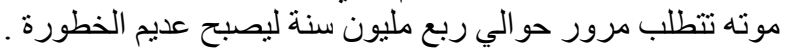

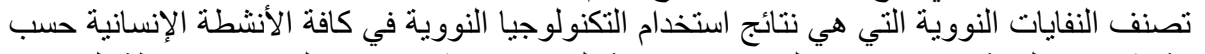

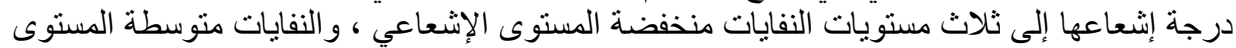
الإثعاعي ، و النفايات عالية المستوى الإثعاعي و هي الأخطر لذلك وجب الإت التعامل معها بحذر شديد عن 
طريق إقامة حواجز و اقية سميكة واتخاذ كل الاحتياطات اللازمة لمنع وقوع تلامس بين هذه النفايات

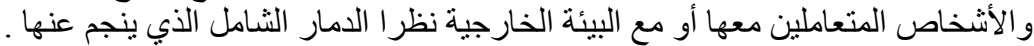

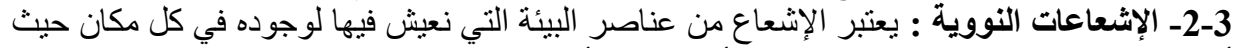

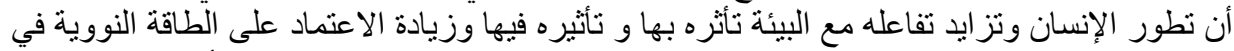

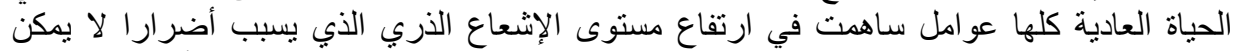

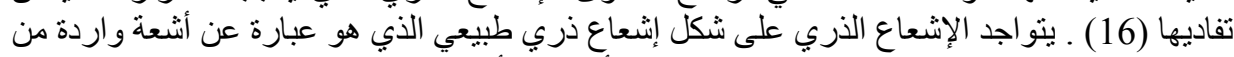

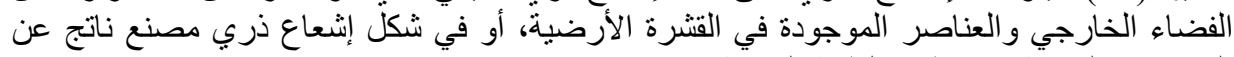

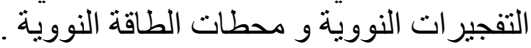

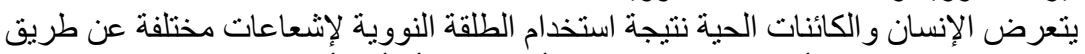

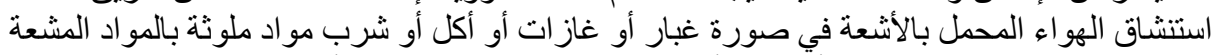

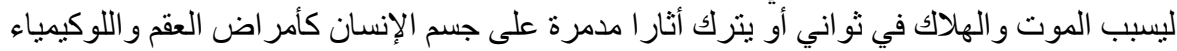

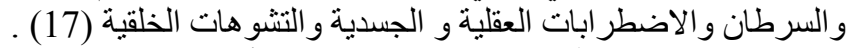

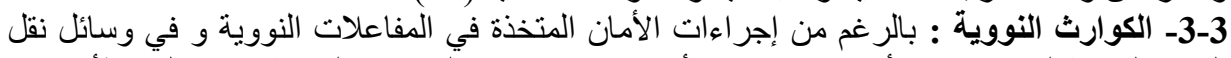

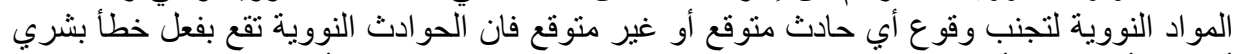

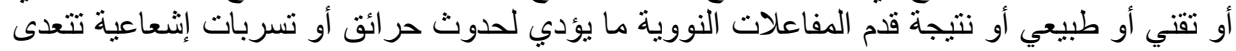

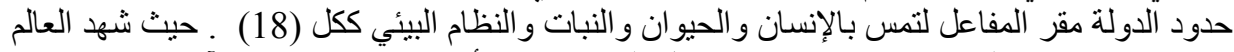

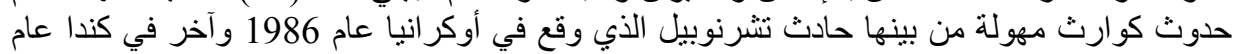

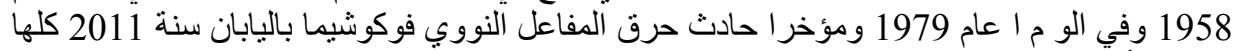

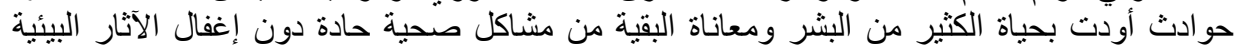

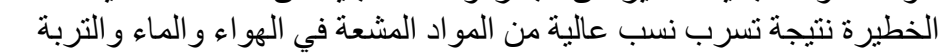

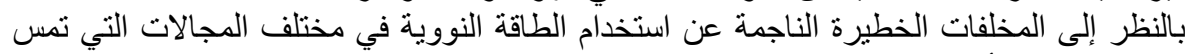

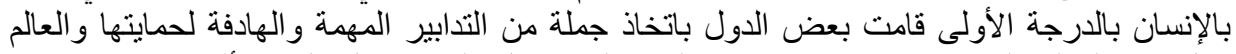

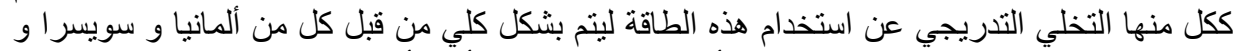

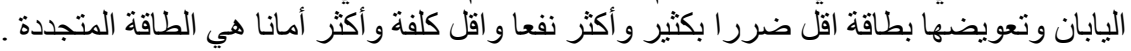

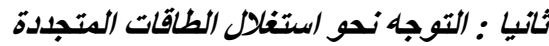

فرضت الطاقة المتجددة نفسها في السنوات الأخيرة كحل بديل للطاقة النووية التي دق المر اقبون التون ناقوس

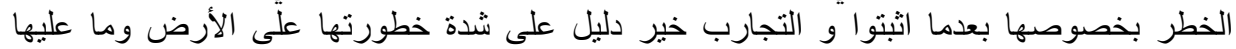

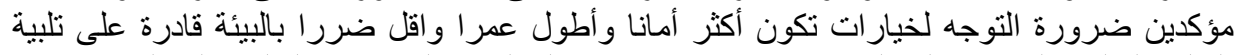

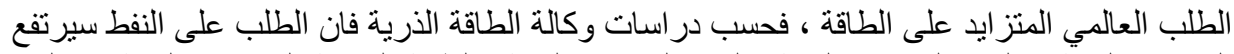
إلى 34 بالمائة و الغاز إلى 24 بالمائة و الفحم إلى 23 بالمائة و الطاقة النووية إلى 4,7 بالمائة في العالم الطي

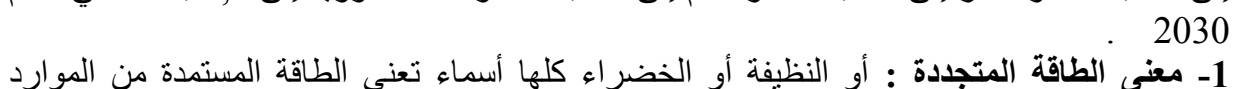

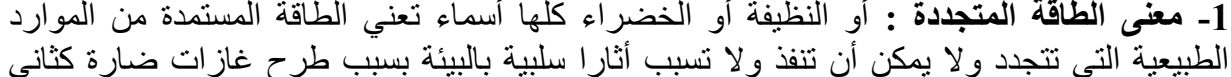

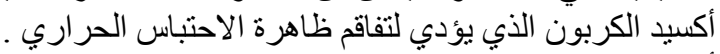

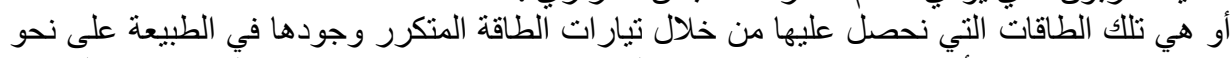

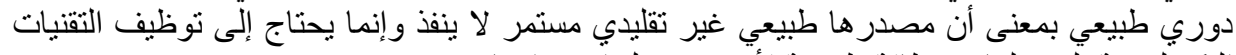

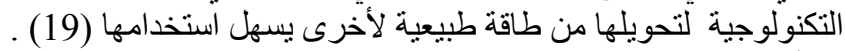

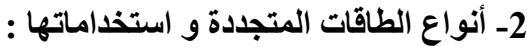

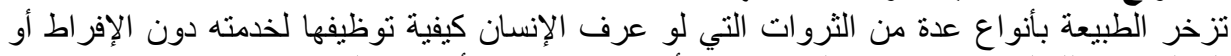

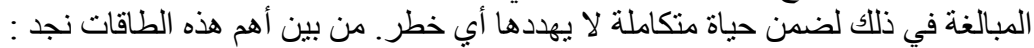

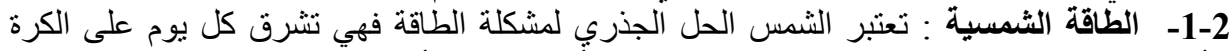

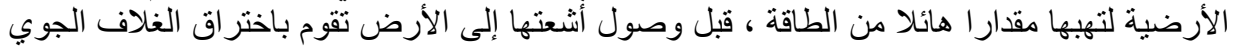




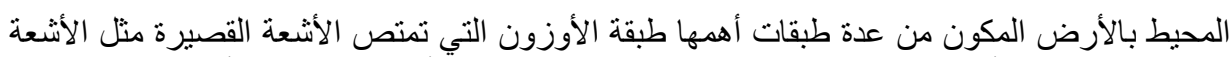

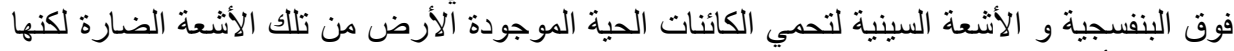

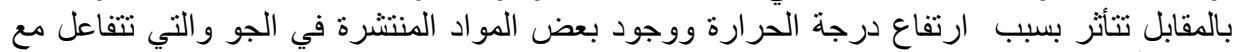

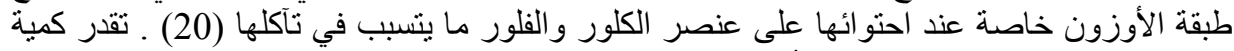

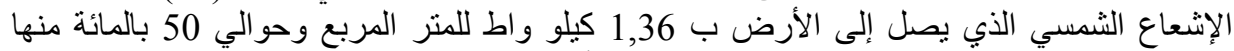
تتعكس في الفضاء و 15 بالمائة تنعكس على سطح الأرض وض و 35 بالمائة يمنص من قبل الهي الهواء و الماء

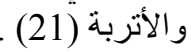

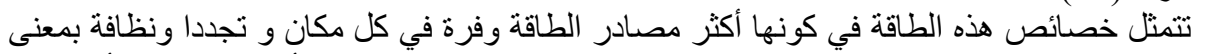

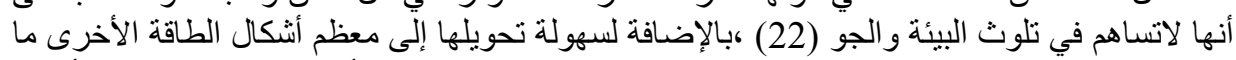

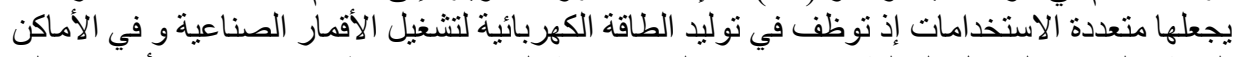

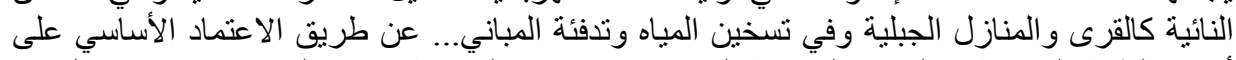

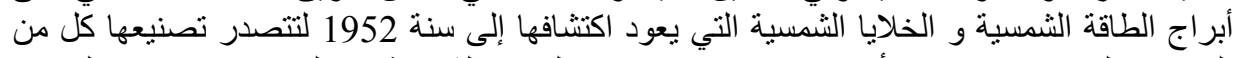

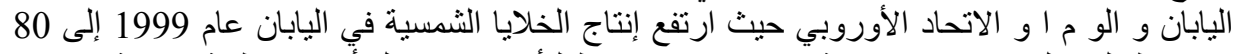

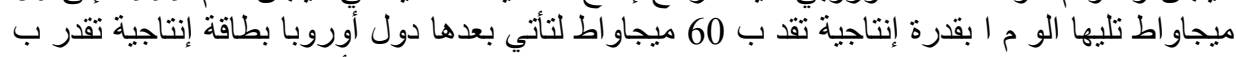

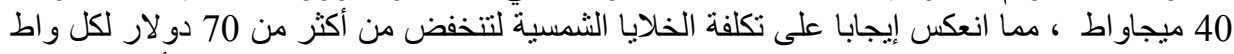

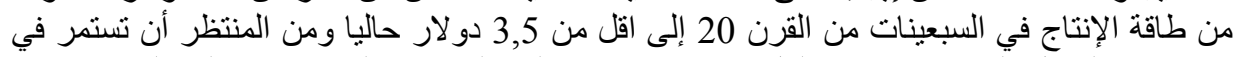

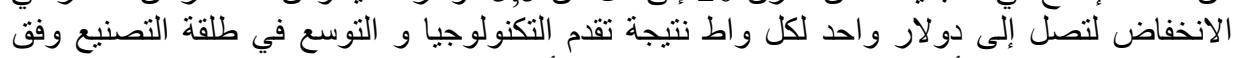

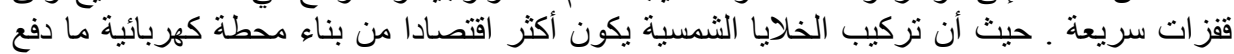

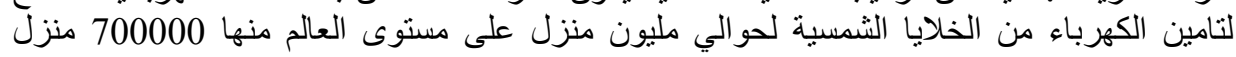

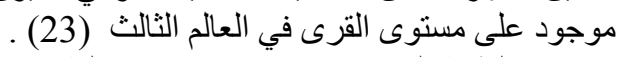

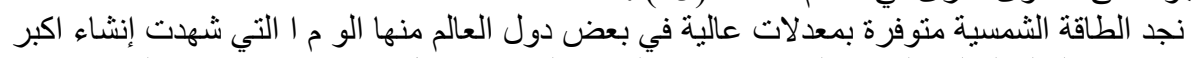

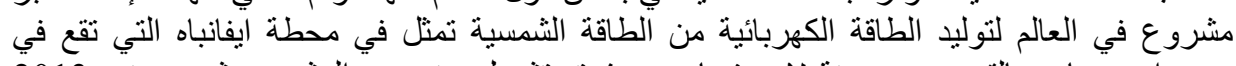

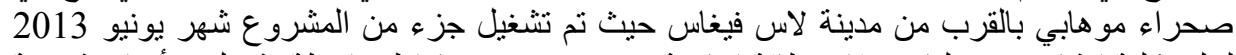

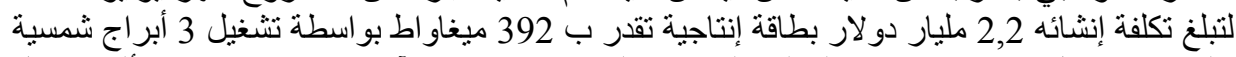

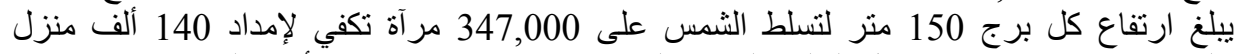

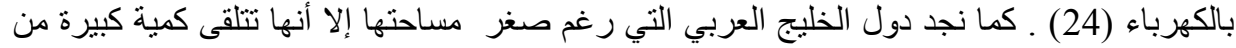

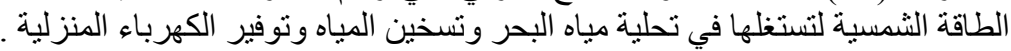

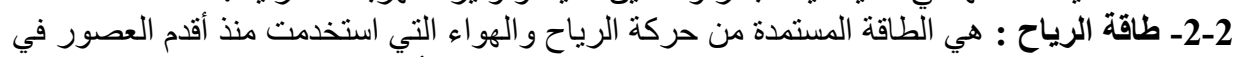

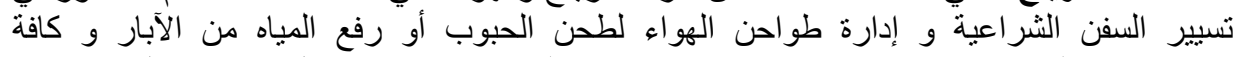

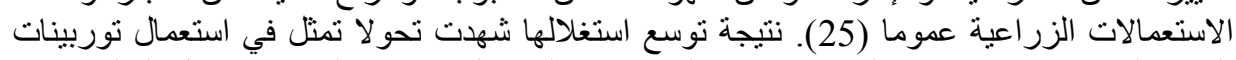

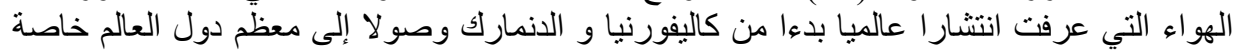

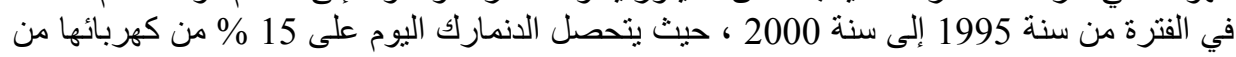

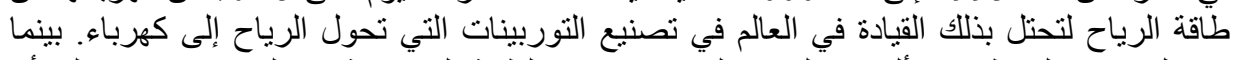

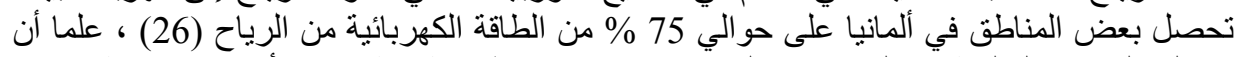
إجمالي القدر ات العالمية من التوربينات بلغت

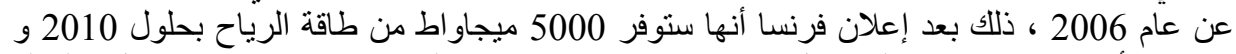

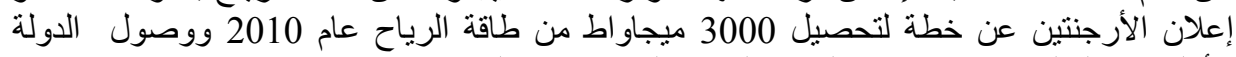

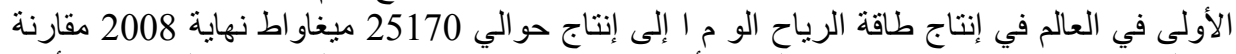

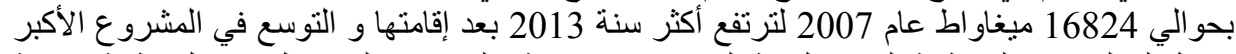

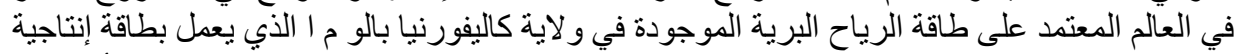

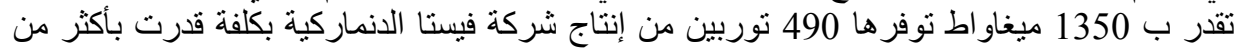
1,2 مليار دو لار (27) ـ بمقابل ذلك قامت بريطانيا بتششين اكبر مزرعة فينا رياح في العالم تانيت التي من 
تجاوزت طاقة إنتاجها للكهرباء الطاقة الإجمالية لباقي دول العالم مع بدئها تشغيل اكبر مزرعة رياح بحرية . تجارن.

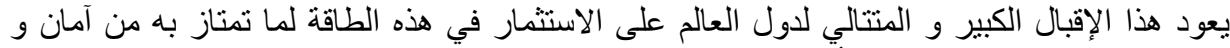

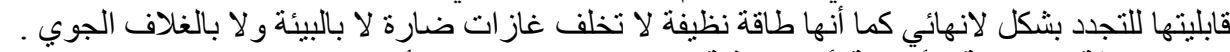

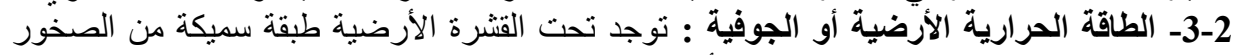

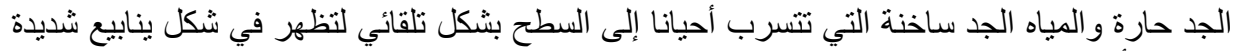

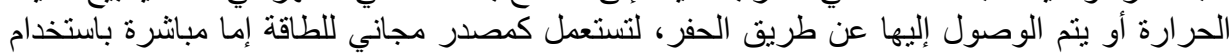

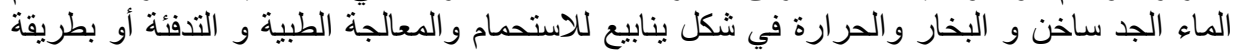
غير مباشرة كوسيلة لتوليد الطاقة الكهربأئية .

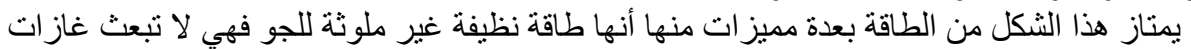

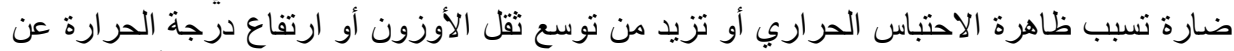

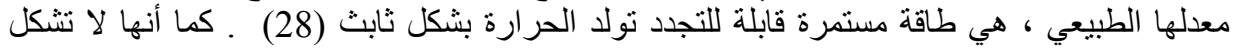

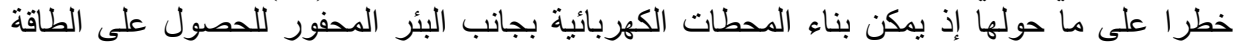

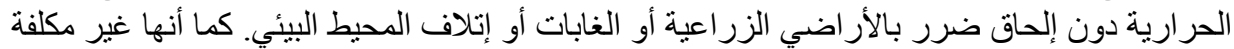

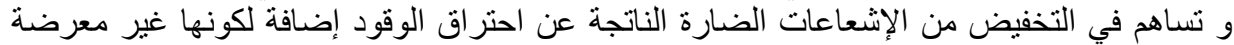

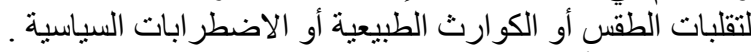

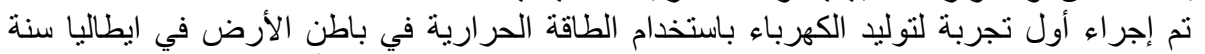

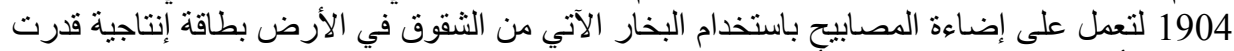

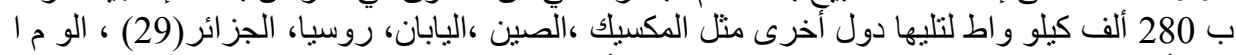

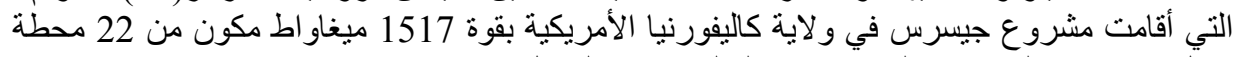

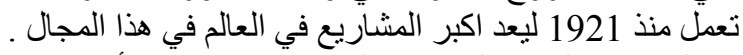

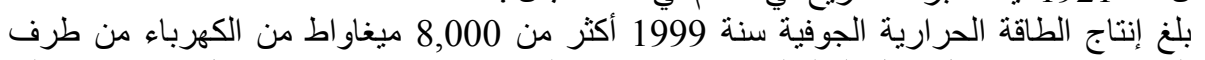

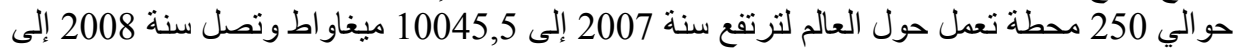
توليد حو الي 10469,7 ميغاو اط حسب إحصائيات وكالة الطاقة الدالئ الدولية .

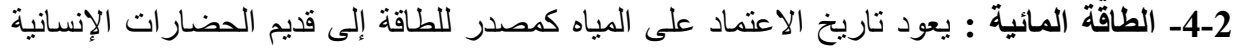

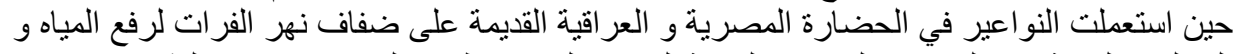

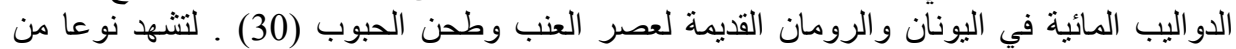

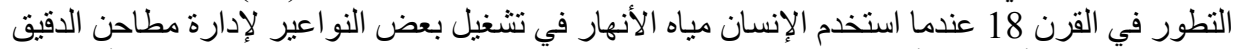

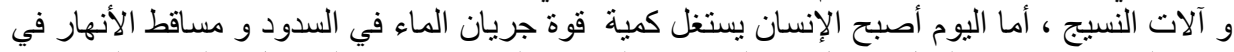

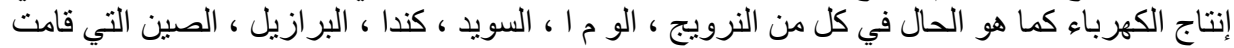

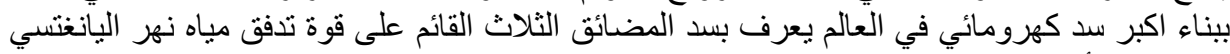

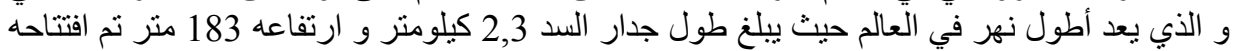

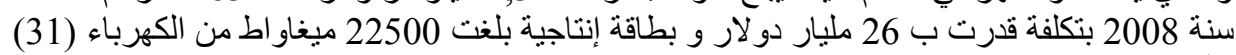

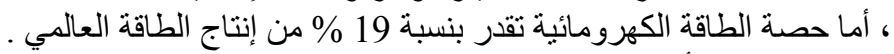

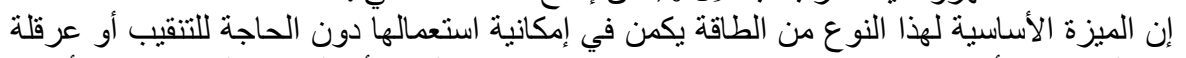

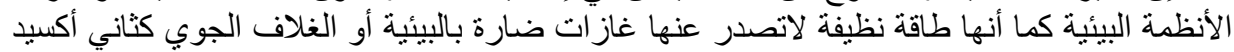

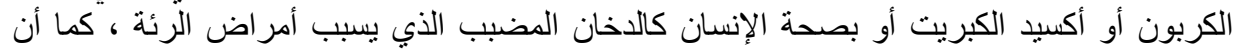

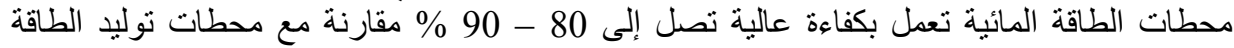

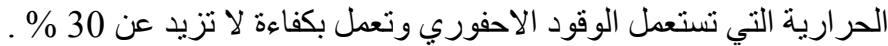

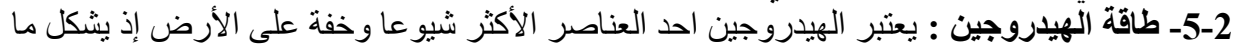

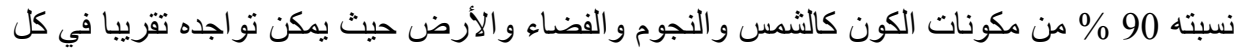
شيء عضوي ، فهو يمثاز بانعدام الرائحة و اللون و الطعم وقبوله الكبير للاشتعال و الاحتر اق دون إنتاج 


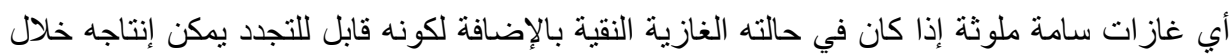

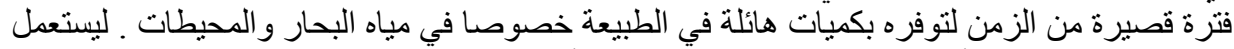

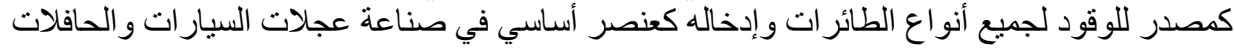

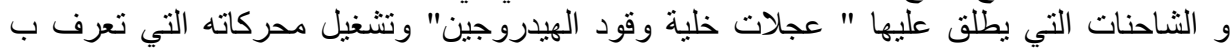

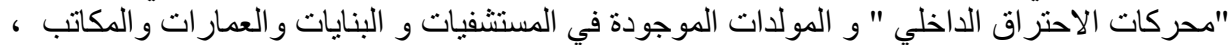

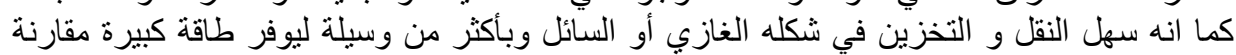

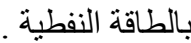

الخاتمة

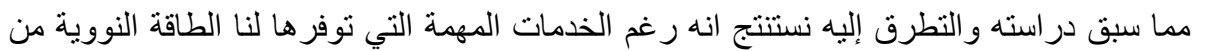

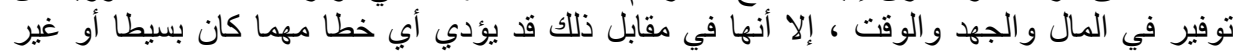

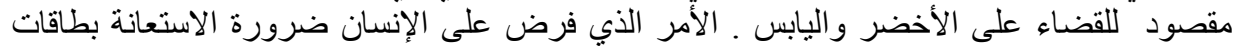

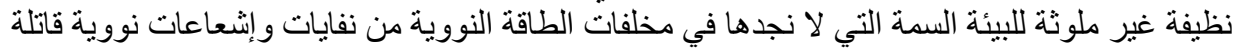

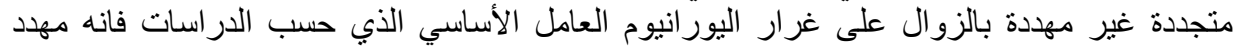

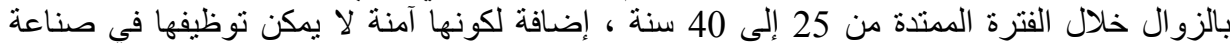

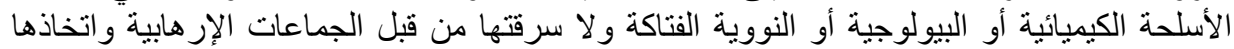

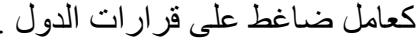

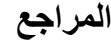

1. Gérard chaliand et Michel jan ; atlas du nucléaire civil et militaire ; payot ,paris , 1993 , p 19

2- محمد عبد الله محمد نعمان ،" ضمانات استخدام الطاقة النووية في الأغراض السلمية" ، جامعة

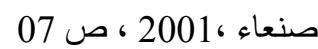

3, Gérard chaliand, op, cit p 21

4ـ د عبد الحكيم طه قنديل ،" النواة والانشطار النووي"، دار الفكر العربي، القاهرة ، الطبعة الأولى،

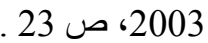

5 , pierre morvan ,nucléaire les chemins de l'uranium , ellipses, paris, 2002, p 28

6- عمر بن عبد الله سعيد البلوشي،" مشروعية أسلحة الدمار الثنامل وفقا لقواعد القانون الدولي"،

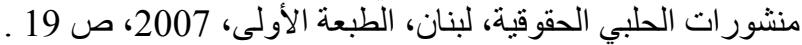

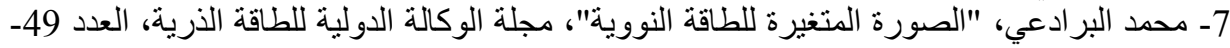

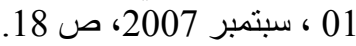

8- ستيف توماس،" اقتصاد الطاقة النووية: آخر المستجدات"، ترجمة رانيا فلفل، مؤسسة هينرش بل

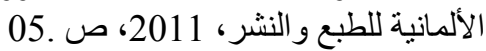
9- محمد كامل محمد،" الطاقة النووية"، مجلة السياسة الدولية، المجلد 41، العدد 165، جويلية 2006،

171. ص ص

10- نصر مهنا،" الذرة و التنمية"، إصدار ات الهيئة العربية للطاقة الذرية، الهجلد 20، العدد الثاني

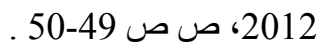

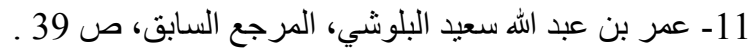

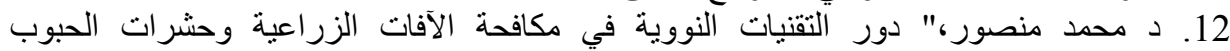

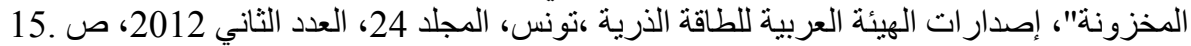

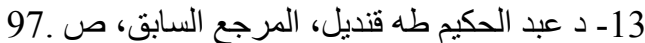

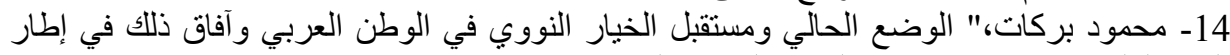

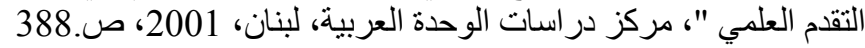


15- مهداوي عبد القادر،" من هيروشيما إلى فوكوشيما: القانون الدولي والاستخدام الأمن للطاقة

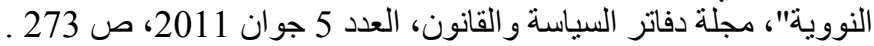

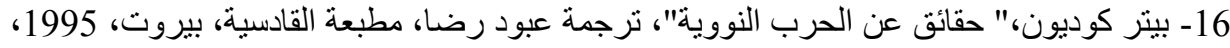
ص 44.

17- عبد الحميد عبد العزيز مشالي،" أخطار التعرض للإشعاع و النظائر المشعة"، مجلة الجندي، العدد 388، ماي 177

18 , barbara viganaux , sureté nucléaire le risque zéro n éxiste pas, alternatives internationales, décembre 2006, p 32,

19- اوشن حنان،" الطاقة البديلة وحماية البيئة في إطار التنمية المستدامة في الجزائر"، موقع مجلة

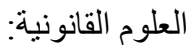

http/ www, marocdroit, com

20- د محمد محمود عمار ،" الطاقة مصادر ها واقتصادياتها"، الطبعة الثانية، مكتبة النهضة المصرية،

القاهرة ص .15 دم

21- مخلفي أمينة،" النفط والطاقات البديلة المتجددة وغير المتجددة"، مجلة الباحث، العدد 09، 2011، 225. ص ص د 212

22- د سمير سعدون مصطفى و آخرون،" الطاقة البديلة مصادرها و استخداماتها"، دار اليازوري

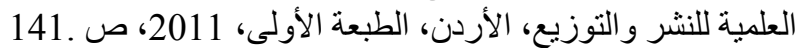

23, christopher flavin and nicholas lenssen, power surge, new york, norton company, 1994, p 154,

24- سهيل سعد،" اكبر مشاريع الطاقة البديلة في العالم"، موقع أخبار الطاقة المتجددة:

http/www, taqat, org/energy/ 1897

25. د راتول محمد وآخرون،" صناعة الطاقات المتجددة بألمانيا وتوجه الجزائر لمشائه الماريع الطاقة

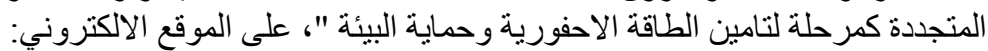
http/manifest,univ- ouargla,dz/documents/archive

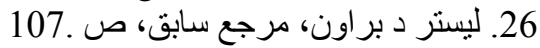

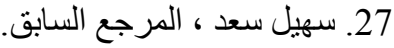

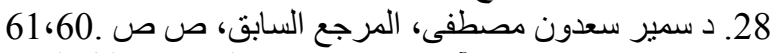

29. كريستوفر فلاقين وآخرون،" طوفان الطاقة : دليل لثورة الطاقة المقبلة"، الدار الدولية للنشر

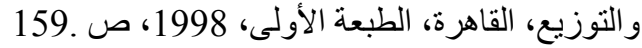

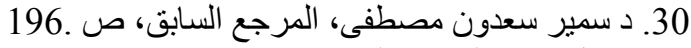

31. سهيل سعد، المرجع السابق. 